\title{
HIGH SPECTRAL RESOLUTION LIDAR BASED ON A POTASSIUM FARADAY DISPERSIVE FILTER FOR DAYTIME TEMPERATURE MEASUREMENT
}

\author{
Makoto Abo*, Phong Pham Le Hoai, Kouki Aruga, Chikao Nagasawa, Yasukuni Shibata \\ Graduate School of System Design, Tokyo Metropolitan University, Tokyo 191-0065, Japan, \\ *Email:abo@tmu.ac.jp
}

\begin{abstract}
In this paper, a new high-spectral-resolution lidar technique is proposed for measuring the profiles of atmospheric temperature in daytime. Based on the theory of high resolution Rayleigh scattering, the feasibility and advantages of using potassium (K) Faraday dispersive optical filters as blocking filters for measuring atmospheric temperature are demonstrated with a numerical simulation. It was found that temperature profiles could be measured within $1 \mathrm{~K}$ error for the height of $9 \mathrm{~km}$ with a 500 $\mathrm{m}$ range resolution in $60 \mathrm{~min}$ by using laser pulses with $1 \mathrm{~mJ} /$ pulse and $1 \mathrm{kHz}$, and a $50 \mathrm{~cm}$ diameter telescope. Furthermore, we are developing compact pulsed laser system for temperature lidar transmitter.
\end{abstract}

\section{INTRODUCTION}

The Earth's weather is driven by the convective processes associated with the atmospheric temperature, water vapor density, and wind, which mostly occur in lower troposphere. Hence, vertical profiles of temperature, humidity, and wind are important data for weather forecasting and prediction of meteorological disaster. Lidar is a preferable instrument to measure these parameters due to the high spatial and temporal resolution profile. The lidar technique for measuring water vapor and wind has been already established. However, a compact temperature lidar for daytime observation has not been realized.

Recently, there are various lidar techniques for temperature measurement including Rayleigh/ Raman Lidar, differential absorption lidar (DIAL), rotational Raman lidar, and high spectral resolution lidar (HSRL). Rayleigh and Raman lidar technique is based on molecular lidar signal that is proportional to molecular number density. This technique requires a hydrostatic equilibrium assumption and an aerosol free altitude region for measuring temperature. DIAL technique is based on the temperature dependence of molecular absorption line. This technique needs a precise tuning laser and three operating wavelengths. Rotational Raman lidar is based on temperature dependence of the population of molecular rotation level. The characteristic of this technique includes narrow linewidth laser, narrow filter, and small scattering cross section.

HSRL technique is fundamentally based on temperature dependence of the Doppler broadening of Rayleigh scattering. This technique required a precise tuning laser and very narrow filter. HSRL performance mostly depends on the stability of filters and laser wavelength. Hence, it is only utilized for measuring nighttime temperature. In this project, we aim at developing a new HSRL system with K Faraday dispersive optical filters to measure atmospheric temperature profile in daytime.

\section{HIGH SPECTRAL RESOLUTION LIDAR FOR TEMPERATURE MEASUREMENT}

2.1 High spectral resolution lidar with absorption filters

The schematic of temperature measurement by the HSRL with absorption cell is shown in Fig. 1. The left figure shows the spectral profile of Rayleigh/Mie backscattering containing a narrow aerosol peak near the laser transmitter frequency and a much broader spectrum of molecular scattering. The backscattering signal passing 
through two absorption cell filters with different temperature results in different residual scattering spectra. Consequently, the atmosphere temperature can be calculated from the ratio of two residual scattering signals [1].

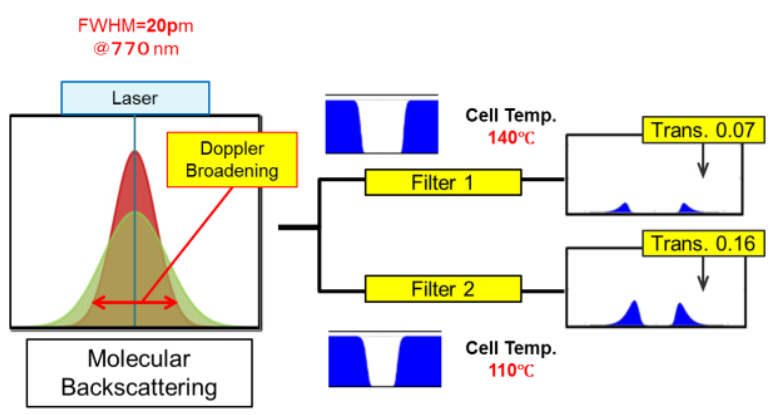

Figure 1. The schematic of temperature measurement by the HSRL with absorption cell.

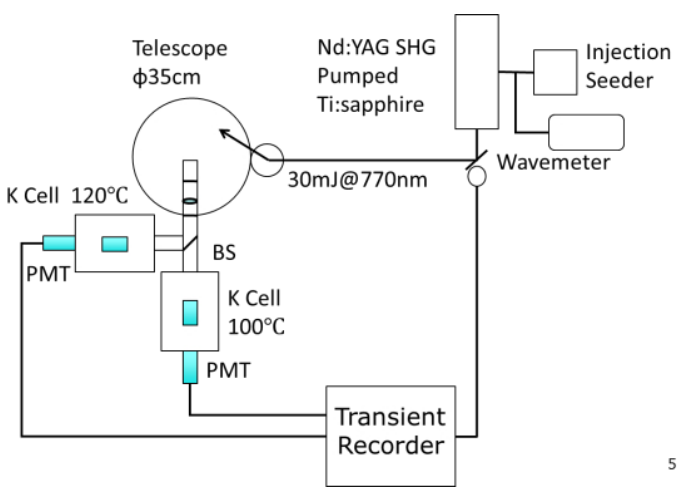

Figure 2. Diagram of HSRL experimental setup with K absorption filters.

Diagram of the HSRL experimental setup with $\mathrm{K}$ absorption filters is shown in Fig. 2. The transmitter utilizes a Nd:YAG- SHG laser as a pump source for Ti: sapphire laser. The output laser with $30 \mathrm{~mJ}$ pulse energy operates at wavelength of $770 \mathrm{~nm}$. The receiver contains a 35 $\mathrm{cm}$ telescope, two $\mathrm{K}$ absorption cells at $100{ }^{\circ} \mathrm{C}$ and $120{ }^{\circ} \mathrm{C}$, photomultiplier tubes (PMT), and a transient recorder. The temperature profile, measured at Hino, Tokyo with $150 \mathrm{~m}$ range resolution is compared to the radiosonde data at Tateno, as shown in Fig. 3. The lidar result coincides with radiosonde data.

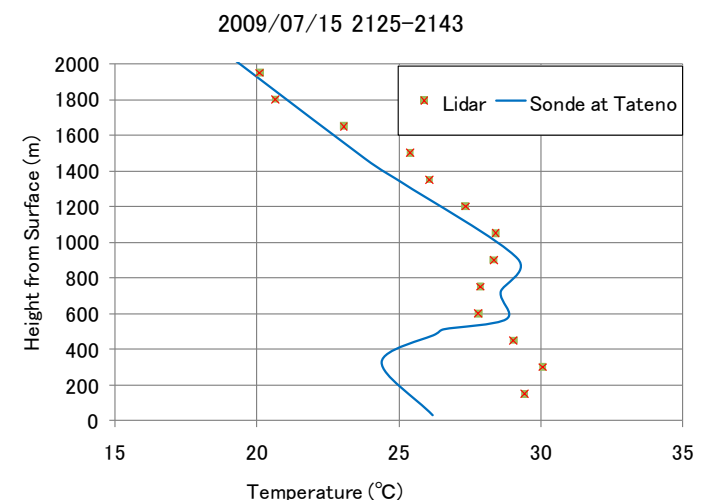

Figure 3. Lidar temperature profile measured at Hino, Tokyo and radiosonde data at Tateno.

\subsection{Faraday Anomalous Dispersion Optical Filter}

A Faraday anomalous dispersion optical filter (FADOF) includes an atomic vapor cell placed between $90^{\circ}$ cross polarizers, as shown in Fig. 4. Magnetic field along the propagation direction induces circular birefringence in the dispersive atomic vapor. Due to the Faraday rotation, only narrow range of wavelengths can be transmitted through filter with high efficiency [2].

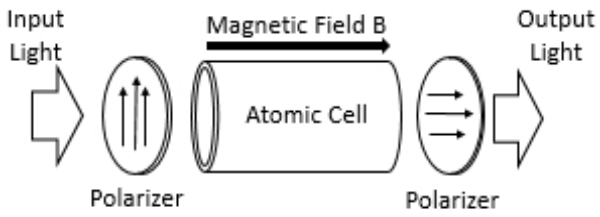

Figure 4. Faraday Anomalous Dispersion Optical Filter.

The transmission profile of FADOF depends on cell temperature, magnetic field, and cell length. Examples of band pass filter and band stop filter are plotted in Fig. 5. The advantage of FADOF is narrow bandwidth and rejected background. While the background rejection of a conventional absorption filter depends on additional interference filter. Hence, we proposed a new HSRL with FADOF for daytime temperature measurement. We also replace a tunable laser of transmitter by compact and simple laser system such as a vapor laser and pulsed tapered semiconductor optical amplifier system. 


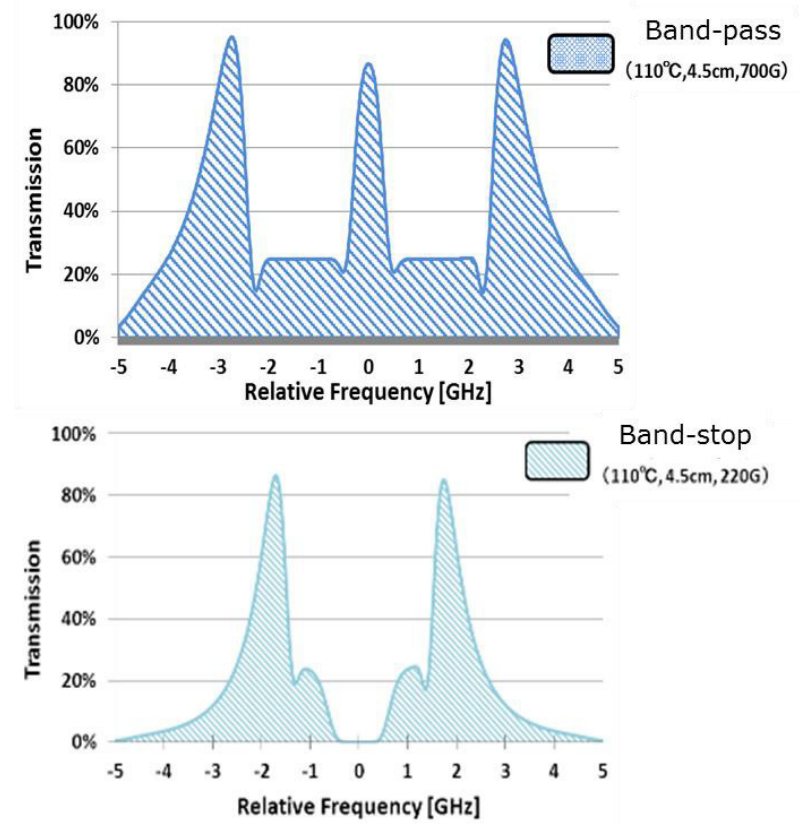

Figure 5. Examples of band pass filter and band stop filter of FADOF.

\subsection{Proposed HSRL with K FADOF}

The schematic of transmission measurement of the K FADOF is shown in Fig. 6. The laser beam from an external cavity diode laser (ECDL) passes through ND filter and Faraday filter, and measured by a photodiode. Measured and calculated transmission profiles of the K FADOF under different conditions are plotted in Fig. 7 The cell temperature is $115^{\circ} \mathrm{C}$, cell length is 7.5 $\mathrm{cm}$, and magnetic field is $330 \mathrm{G}$ and $115 \mathrm{G}$ respectively. Consequently, the experimental results agree well with theoretical results.

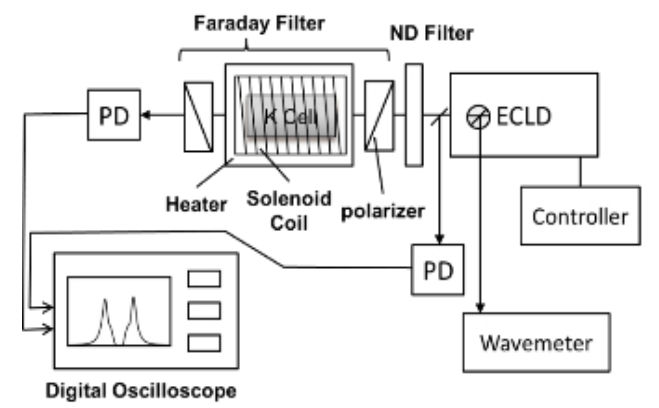

Figure 6. Schematic of transmission measurement of the K FADOF.

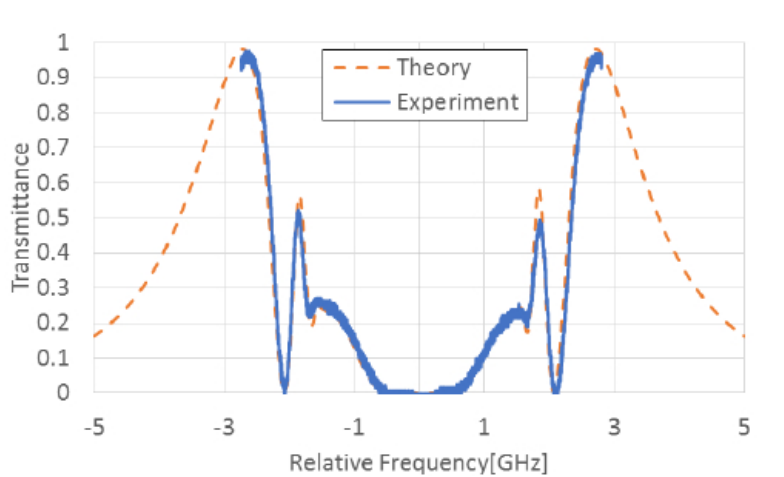

(a)

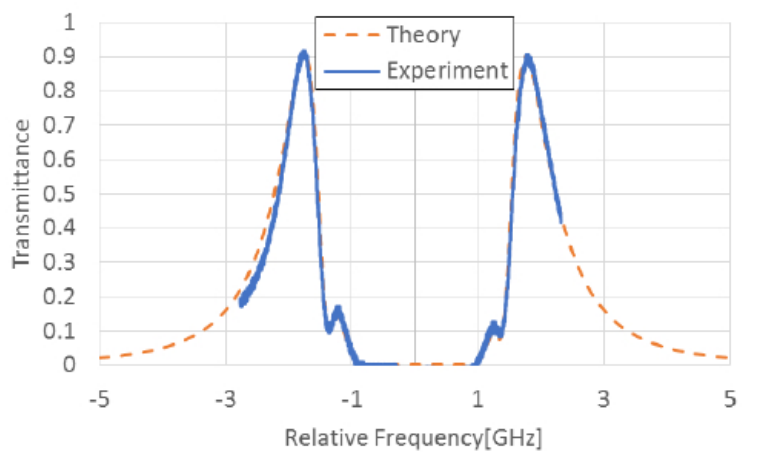

(b)

Figure 7. Measured (solid line) and calculated (dashed line) transmission profiles of the K FADOF. The cell temperature is $115^{\circ} \mathrm{C}$, cell length is $7.5 \mathrm{~cm}$, and magnetic field is (a) $330 \mathrm{G}$ and (b) $115 \mathrm{G}$.

The HSRL parameters for temperature error simulation are given in Table 1. A laser beam with pulse energy of $1 \mathrm{~mJ}$ and repetition rate of $1 \mathrm{kHz}$ is transmitted to low altitude region from ground to $6 \mathrm{~km}$ and high altitude region from $6 \mathrm{~km}$ to 20 $\mathrm{km}$. The receiver contains $20 \mathrm{~cm} / 50 \mathrm{~cm}$ telescope for low / high altitude system. The K FADOF specifications include cell length of 7.5 $\mathrm{cm}$, cell temperature of $115^{\circ} \mathrm{C}$, and magnetic field of $115 \mathrm{G} / 330 \mathrm{G}$ for low / high altitude region.

Table 1. The HSRL parameters for simulation.

\begin{tabular}{|l|l|l|}
\hline & Low Altitude & High Altitude \\
\hline Target Altitude & $0 \mathrm{~km} \sim 6 \mathrm{~km}$ & $6 \mathrm{~km} \sim 20 \mathrm{~km}$ \\
\hline Pulse energy & $1 \mathrm{~mJ}$ \\
\hline Repetition rate & $1 \mathrm{kHz}$ \\
\hline Telescope Aperture & $20 \mathrm{~cm}$ & $50 \mathrm{~cm}$ \\
\hline Range Resolution & $200 \mathrm{~m}$ & $500 \mathrm{~m}$ \\
\hline Accumulation time & $10 \mathrm{~min}$ & $60 \mathrm{~min}$ \\
\hline
\end{tabular}

The simulated temperature errors of conventional HSRL and proposed HSRL are shown in Fig. 8. Our proposed HSRL using K FADOF shows 
smaller error than the conventional HSRL using absorption filter during daytime and nighttime as well as low and high altitude region. For low altitude system, the proposed HSRL can measure the temperature up to $3 \mathrm{~km}$ within $1 \mathrm{~K}$ error for daytime with a $200 \mathrm{~m}$ range resolution in 10 minutes. For high altitude system, the proposed HSRL can measure the temperature up to $9 \mathrm{~km}$ within $1 \mathrm{~K}$ error for daytime with a $500 \mathrm{~m}$ range resolution in 60 minutes. Hence, the proposed HSRL is appropriate for daytime temperature measurement.

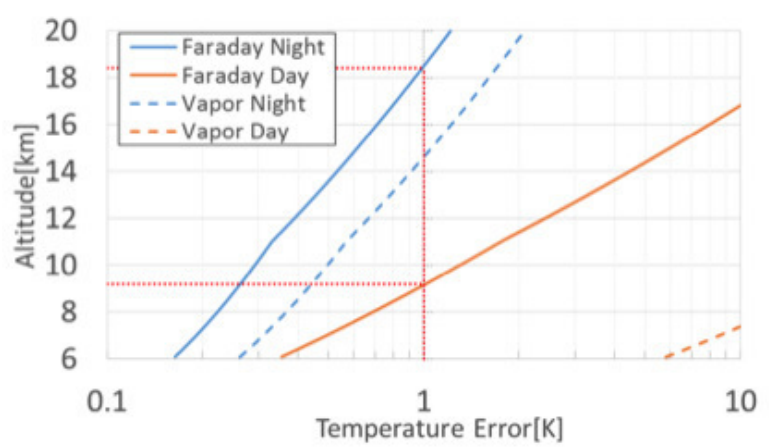

(a)

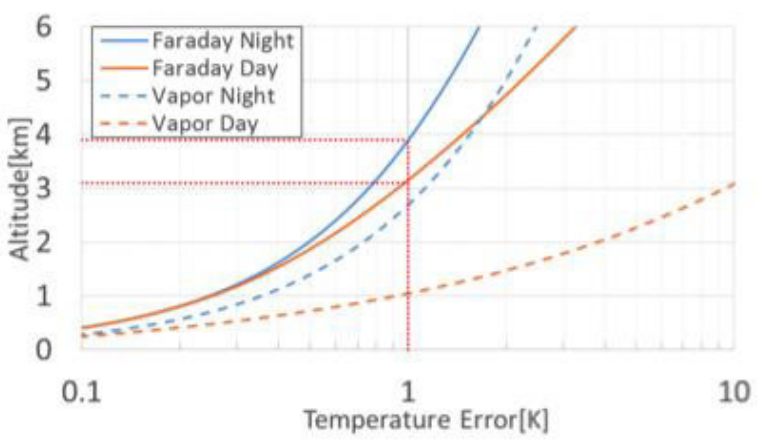

(b)

Figure 8. The simulated temperature errors of absorption-filter HSRL (dashed line) and proposed Faraday-filter HSRL (solid line) for nighttime (blue) and daytime (red) observations with (a) high altitude system and (b) low altitude system.

\section{TRANSMITTER}

We are developing two type of transmitter for the compact and simple temperature HSRL. One laser is a pulsed $\mathrm{K}$ vapor laser. The $\mathrm{K}$ vapor laser can easily realize narrow-linewidth and precise tuning. A simple configuration for experimental setup is shown in Fig. 9. A pumped laser beam passes through haft wave plate, a polarized beam splitter and injected to a $K$ vapor cell in oven with $A R$ coating. A high reflector mirror placed behind the
$\mathrm{K}$ cell to reflect the laser beam back. Then the output laser beam directs to the polarized beam splitter and to be transmitted to the air.

Another laser is tapered amplifier diode system by seeding with continuous-wave DBR laser [3]. The DBR laser is easily tuned to $\mathrm{K}$ resonance line using wavelength-locking system, and tapered amplifier diode can provide high repletion rate pulse laser.

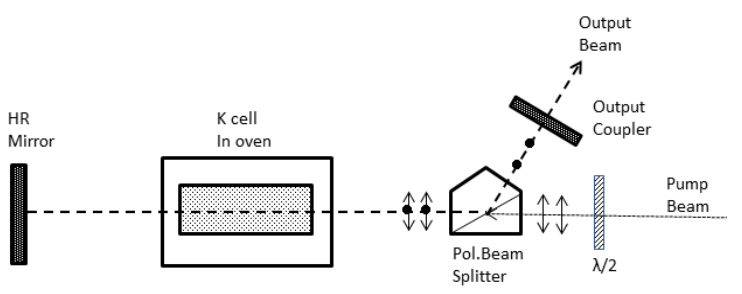

Figure 9. Experimental Setup of a Pulsed Laser Pumped K Vapor Laser

\section{CONCLUSIONS}

In this paper, we propose a new high-spectralresolution lidar for measuring the profiles of atmospheric temperature in daytime. The $\mathrm{K}$ Faraday dispersive optical filters act as blocking filters for measuring atmospheric temperature. The simulation results show that the proposed HSRL is suited for daytime temperature measurement. Furthermore, we are developing compact pulsed laser system for temperature lidar transmitter.

\section{ACKNOWLEDGEMENT}

This work was supported by JSPS KAKENHI Grant Number 26550014.

\section{REFERENCES}

[1] Shimizu, H., Lee, S. A., and She, C. Y., 1983: High spectral resolution lidar system with atomic blocking filters for measuring atmospheric parameters, Appl. Opt., 22, 1373-1381.

[2] Zhang, Y., Jia, X., Ma, Z., Wang, Q., 2001: Optical filtering characteristic of potassium Faraday optical filter, IEEE J. of Quantum Electronics, 37, 372-375.

[3] Xiong, Y., Murphy, S., Carlsten, J. L., Repasky, K., S., 2006: Design and characteristics of a tapered amplifier diode system by seeding with continuous-wave and mode-locked external cavity diode laser, Opt. Eng. 45(12), 124205. 